\title{
ON THE RECURSIVE SEQUENCE
}

\author{
E. CAMOUZIS, R. DEVAULT, AND G. PAPASCHINOPOULOS
}

Received 12 January 2004 and in revised form 29 May 2004

Our aim in this paper is to investigate the boundedness, global asymptotic stability, and periodic character of solutions of the difference equation $x_{n+1}=\left(\gamma x_{n-1}+\delta x_{n-2}\right) /\left(x_{n}+\right.$ $\left.x_{n-2}\right), n=0,1, \ldots$, where the parameters $\gamma$ and $\delta$ and the initial conditions are positive real numbers.

\section{Introduction}

Using an appropriate change of variables, we have that the recursive sequence $x_{n+1}=$ $\left(\gamma x_{n-1}+\delta x_{n-2}\right) /\left(x_{n}+x_{n-2}\right)$ is equivalent to the difference equation

$$
x_{n+1}=\frac{\gamma x_{n-1}+x_{n-2}}{x_{n}+x_{n-2}}, \quad n=0,1, \ldots
$$

For all values of the parameter $\gamma,(1.1)$ has a unique positive equilibrium $\bar{x}=(\gamma+1) / 2$. When $0<\gamma<1$, the positive equilibrium $\bar{x}$ is locally asymptotically stable. In the case where $\gamma=1$, the characteristic equation of the linearized equation about the positive equilibrium $\bar{x}=1$ has three eigenvalues, one of which is -1 , and the other two are 0 and $1 / 2$. In addition, when $\gamma=1,(1.1)$ possesses infinitely many period-two solutions of the form $\{a, a /(2 a-1), a, a /(2 a-1), \ldots\}$ for all $a>1 / 2$. When $\gamma>1$, the equilibrium $\bar{x}$ is hyperbolic.

The investigation of (1.1) has been posed as an open problem in [1,2]. In this paper, we will show that when $0<\gamma<1$, the interval $[\gamma, 1]$ is an invariant interval for $(1.1)$ and that every solution of (1.1) falling into this interval converges to the positive equilibrium $\bar{x}$. Furthermore, we will show that when $\gamma=1$, every positive solution $\left\{x_{n}\right\}_{n=-2}^{\infty}$ of (1.1) which is eventually bounded from below by $1 / 2$ converges to a (not necessarily prime) period-two solution. Finally, when $\gamma>1$, we will prove that (1.1) possesses unbounded solutions. We also pose some open questions for (1.1).

We say that a solution $\left\{x_{n}\right\}_{n=-k}^{\infty}$ of a difference equation is bounded and persists if there exist positive constants $P$ and $Q$ such that

$$
P \leq x_{n} \leq Q \text { for } n=-k,-k+1, \ldots
$$




\section{The case $\gamma<1$}

In this section, we find conditions under which solutions of (1.1) converge to the positive equilibrium $\bar{x}$.

Theorem 2.1. Suppose that $0<\gamma<1$ and $\left\{x_{n}\right\}_{n=-2}^{\infty}$ is a solution of (1.1) for which there exists $N \geq 0$ such that $x_{N-2}, x_{N-1}$, and $x_{N} \in[\gamma, 1]$. Then

$$
\lim _{n \rightarrow \infty} x_{n}=\frac{\gamma+1}{2}
$$

Proof. If there exists $N \geq 0$ such that $x_{N-2}, x_{N-1}$, and $x_{N} \in[\gamma, 1]$, then

$$
\gamma=\frac{\gamma^{2}+\gamma}{1+\gamma} \leq \frac{\gamma^{2}+x_{N-2}}{1+x_{N-2}} \leq x_{N+1}=\frac{\gamma x_{N-1}+x_{N-2}}{x_{N}+x_{N-2}} \leq \frac{\gamma+x_{N-2}}{\gamma+x_{N-2}}=1
$$

Thus by induction we have

$$
x_{n} \in[\gamma, 1] \quad \forall n \geq N-2 .
$$

Now let

$$
I=\liminf _{n \rightarrow \infty} x_{n}, \quad S=\limsup _{n \rightarrow \infty} x_{n}
$$

Then $\gamma \leq I \leq S \leq 1$ and there exist two solutions $\left\{I_{n}\right\}_{n=-\infty}^{\infty}$ and $\left\{S_{n}\right\}_{n=-\infty}^{\infty}$ of (1.1) such that $I_{0}=I, S_{0}=S$, and $I_{n}, S_{n} \in[I, S]$ for all $n \in Z$. Then

$$
S=S_{0}=\frac{\gamma S_{-2}+S_{-3}}{S_{-1}+S_{-3}} \leq \frac{\gamma S+S_{-3}}{I+S_{-3}} \leq \frac{\gamma S+S}{I+S}
$$

and so $I+S \leq \gamma+1$. Also

$$
I=I_{0}=\frac{\gamma I_{-2}+I_{-3}}{I_{-1}+I_{-3}} \geq \frac{\gamma I+I_{-3}}{S+I_{-3}} \geq \frac{\gamma I+I}{S+I}
$$

which implies that $I+S \geq \gamma+1$ and so $I+S=\gamma+1$. If $S_{-2}<S$ or $S_{-1}>I$, then

$$
S=S_{0}=\frac{\gamma S_{-2}+S_{-3}}{S_{-1}+S_{-3}}<\frac{\gamma S+S_{-3}}{I+S_{-3}} \leq \frac{\gamma S+S}{I+S}
$$

and so $I+S<\gamma+1$, which is a contradiction. Therefore, $S_{-2}=S$ and $S_{-1}=I$. Similarly, $S_{-4}=S$ and $S_{-3}=I$. Thus

$$
S=S_{0}=\frac{\gamma S_{-2}+S_{-3}}{S_{-1}+S_{-3}}=\frac{\gamma S+I}{I+I}
$$


which implies that $2 I S=\gamma S+I$. Similarly, we can show that $I_{-1}=S, I_{-2}=I$, and $I_{-3}=S$, and so we have

$$
I=I_{0}=\frac{\gamma I_{-2}+I_{-3}}{I_{-1}+I_{-3}}=\frac{\gamma I+S}{S+S}
$$

which implies that $2 I S=\gamma I+S$. Therefore, since $0<\gamma<1, I=S$ and the proof is complete.

We end this section with the following open problem.

Open problem 2.2. Prove that when $0<\gamma<1$, the interval $[\gamma, 1]$ is globally attractive, thus showing that the positive equilibrium $\bar{x}$ of (1.1) is globally asymptotically stable.

\section{The case $\gamma=1$}

In this section, we show that when $\gamma=1$, every positive solution $\left\{x_{n}\right\}_{n=-2}^{\infty}$ of (1.1) which is eventually bounded from below by $1 / 2$ converges to a (not necessarily prime) periodtwo solution.

If $\gamma=1$, then (1.1) becomes

$$
x_{n+1}=\frac{x_{n-1}+x_{n-2}}{x_{n}+x_{n-2}}, \quad n=0,1, \ldots
$$

The following lemma provides an important identity, the proof of which follows from straightforward calculations using (3.1).

Lemma 3.1. Let $\left\{x_{n}\right\}_{n=-2}^{\infty}$ be a positive solution of (1.1). Then for $n \geq 2$,

$$
\left(x_{n+1}-x_{n-1}\right)\left(x_{n}+x_{n-2}\right)=x_{n-1}\left(x_{n}-x_{n-2}\right)+\frac{\left(x_{n-1}-x_{n-3}\right)\left(x_{n-1}-x_{n-2}\right)}{x_{n-1}+x_{n-3}} .
$$

LEMMA 3.2. Every nonoscillatory solution of (3.1) converges monotonically to the positive equilibrium $\bar{x}=1$.

Proof. Let $\left\{x_{n}\right\}_{n=-2}^{\infty}$ be a solution of (3.1) and suppose that there exists an integer $N \geq-2$ such that

$$
x_{n}<1, \quad n=N, N+1, \ldots
$$

The case where the solution is eventually greater than or equal to $\bar{x}=1$ is similar and will be omitted. Using (3.1), we have

$$
x_{n+1}>x_{n}, \quad n=N-2, N-1, \ldots,
$$

and so the solution $\left\{x_{n}\right\}_{n=-2}^{\infty}$ converges to the positive equilibrium $\bar{x}=1$. The proof is complete.

Lemma 3.3. Let $\left\{x_{n}\right\}_{n=-2}^{\infty}$ be a positive solution of (3.1) for which there exists $N \geq-1$ such that

$$
x_{N}<1, \quad x_{N+1} \geq 1 .
$$


Then for all $n \geq 1$,

$$
x_{2 n+N}<1, \quad x_{2 n+N+1} \geq 1 .
$$

Proof. Using (3.1) and in view of (3.5), we have

$$
x_{N+2}=\frac{x_{N}+x_{N-1}}{x_{N+1}+x_{N-1}}<1<\frac{x_{N+1}+x_{N}}{x_{N+2}+x_{N}}=x_{N+3} .
$$

The proof of (3.6) follows by induction.

Lemma 3.4. Let $\left\{x_{n}\right\}_{n=-2}^{\infty}$ be a positive oscillatory solution of (3.1) which is bounded and persists. Then

$$
2 I S=I+S, \quad I \in\left(\frac{1}{2}, 1\right], S \in[1, \infty),
$$

where (2.4) holds.

Proof. There exist subsequences of $\left\{x_{n}\right\}_{n=-2}^{\infty}$, namely, $\left\{x_{n_{i}+k}\right\}_{i=1}^{\infty}, k=-2,-1,0,1$ such that

$$
\lim _{i \rightarrow \infty} x_{n_{i}+k}=l_{k}
$$

In addition,

$$
x_{n_{i}+k}>1, \quad k=-1,1, \quad x_{n_{i}+m}<1, \quad m=-2,0
$$

for all $i$ and

$$
S=l_{1}
$$

It follows that

$$
S, l_{-1} \in[1, \infty), \quad I, l_{-2}, l_{0} \in(0,1]
$$

From (3.1), we have

$$
S=\frac{l_{-1}+l_{-2}}{l_{0}+l_{-2}} \leq \frac{S+I}{2 I}
$$

and so

$$
2 S I \leq S+I
$$

On the other hand, there exist subsequences $\left\{x_{m_{j}+k}\right\}_{j=1}^{\infty}, k=-2,-1,0,1,2$ such that

$$
\lim _{j \rightarrow \infty} x_{m_{j}+k}=m_{k}
$$

such that, for all $j$,

$$
x_{m_{j}+k}>1, \quad k=-1,1, \quad x_{m_{j}+k}<1, \quad k=-2,0,2
$$


and also

$$
I=m_{2}
$$

Hence,

$$
S, m_{-1}, m_{1} \in[1, \infty), \quad I, m_{-2}, m_{0} \in(0,1] .
$$

If $m_{0}<m_{-2}$ and $m_{1}>m_{-1}$, then in view of (3.2) we have $I>m_{0}$, which is a contradiction.

If $m_{0}<m_{-2}$ and $m_{1} \leq m_{-1}$, from (3.1) we have

$$
I=\frac{m_{0}+m_{-1}}{m_{1}+m_{-1}} \geq \frac{m_{0}+m_{-1}}{2 m_{-1}} \geq \frac{I+S}{2 S} .
$$

If $m_{0} \geq m_{-2}$, using (3.1) we have

$$
I=\frac{\left(m_{0}+m_{-1}\right)\left(m_{0}+m_{-2}\right)}{m_{-1}+m_{-2}+m_{-1}\left(m_{0}+m_{-2}\right)} \geq \frac{(I+S) 2 I}{S+I+2 I S} .
$$

Hence,

$$
2 S I=S+I
$$

To prove that $I>1 / 2$, assume for the sake of contradiction that $I \leq 1 / 2$. Then, since $2 S I=$ $S+I$, we have

$$
S \geq S+I
$$

and so $I \leq 0$, which is a contradiction. Thus $I>1 / 2$. Clearly $S \geq 1$. The proof is complete.

THEOREM 3.5. Let $\left\{x_{n}\right\}_{n=-2}^{\infty}$ be a positive oscillatory solution of (3.1) for which there exists $N \geq 0$ such that $x_{N-1}>1>x_{N-2}, x_{N}>1 / 2$. Then there exists $c \in(1 / 2,1)$ such that

$$
c<x_{n}<\frac{c}{2 c-1}, \quad n=N-2, N-1, \ldots,
$$

and so the solution is bounded and persists.

Proof. Suppose that there exists $N \geq 0$ such that

$$
\frac{1}{2}<x_{N-2}, x_{N}<1<x_{N-1}
$$

Then there exists $c \in(1 / 2,1)$ such that

$$
c<x_{N-2}, x_{N}<1<x_{N-1}<\frac{c}{2 c-1} .
$$

We will show that $x_{N+1}, x_{N+2} \in(c, c /(2 c-1))$. The proof then follows inductively. From (3.1), we have

$$
1<x_{N+1}=\frac{x_{N-1}+x_{N-2}}{x_{N}+x_{N-2}}<\frac{c+c /(2 c-1)}{2 c}=\frac{c}{2 c-1} .
$$


Case 1. $x_{N+1} \leq x_{N-1}$. Then

$$
1>x_{N+2}=\frac{x_{N}+x_{N-1}}{x_{N+1}+x_{N-1}} \geq \frac{x_{N}+x_{N-1}}{2 x_{N-1}}>\frac{c+c /(2 c-1)}{2 c /(2 c-1)}=c .
$$

Case 2. $x_{N+1}>x_{N-1}, x_{N} \leq x_{N-2}$. In view of (3.2), we have

$$
1>x_{N+2}>x_{N}>c
$$

Case 3. $x_{N+1}>x_{N-1}, x_{N}>x_{N-2}$. From (3.1), we have

$$
1>x_{N+2}=\frac{\left(x_{N}+x_{N-1}\right)\left(x_{N}+x_{N-2}\right)}{x_{N-1}+x_{N-2}+x_{N-1}\left(x_{N}+x_{N-2}\right)}>\frac{(c+c /(2 c-1))(2 c)}{c /(2 c-1)+c+(c /(2 c-1))(2 c)}=c .
$$

LEMma 3.6. Let $\left\{x_{n}\right\}_{n=-2}^{\infty}$ be a positive oscillatory solution of (3.1) for which there exists $N \geq 0$ such that $x_{N-1}>1>x_{N-2}, x_{N}>1 / 2$. Let (2.4) hold and let $\left\{x_{n_{i}}\right\}_{i=1}^{\infty}$ be a subsequence of $\left\{x_{n}\right\}_{n=-2}^{\infty}$ such that

$$
\lim _{i \rightarrow \infty} x_{n_{i}}=L \in\{I, S\}
$$

Then

$$
\lim _{i \rightarrow \infty} x_{n_{i}-1}=\frac{L}{2 L-1}, \quad \lim _{i \rightarrow \infty} x_{n_{i}-2}=L
$$

Proof. Let $L_{-2}$ be any accumulation point for $\left\{x_{n_{i}-2}\right\}_{i=1}^{\infty}$. There exists a further subsequence $\left\{n_{i_{s}}\right\}_{s=1}^{\infty}$ of $\left\{n_{i}\right\}_{i=1}^{\infty}$ such that

$$
\lim _{s \rightarrow \infty} x_{n_{i s}+k}=L_{k}, \quad k=-4,-3,-2,-1,0 .
$$

In addition,

$$
\lim _{s \rightarrow \infty} x_{n_{i s}}=L
$$

Assume that $L=S$, and for all $s$,

$$
x_{n_{i s}+k}>1, \quad k=-2,0, \quad x_{n_{i s}+m}<1, \quad m=-1,-3 .
$$

Then $S, L_{-2} \geq 1 \geq L_{-1}, L_{-3}$. From (3.1), we have

$$
S=\frac{L_{-2}+L_{-3}}{L_{-1}+L_{-3}} \leq \frac{S+I}{L_{-1}+I}=\frac{2 S I}{L_{-1}+I}
$$

which implies that $L_{-1}=I$ and also $L_{-2}=S$. On the other hand if $L=I$, without loss of generality we assume that for all $s=0,1, \ldots$,

$$
x_{n_{i s}+k}>1, \quad k=-1,3, \quad x_{n_{i s}+m}<1, \quad m=-2,0 .
$$

We consider the following two cases. 
Case 1. $L_{-1} \leq L_{-3}$. Then

$$
I=\frac{L_{-2}+L_{-3}}{L_{-1}+L_{-3}} \geq \frac{L_{-1}+L_{-2}}{2 L_{-1}} \geq \frac{S+I}{2 S}=I
$$

and so $L_{-1}=L_{-2} /(2 I-1) \geq I /(2 I-1)=S$ which implies that $L_{-1}=S$ and $L_{-2}=I$.

Case 2. $L_{-1}>L_{-3}$. If $L_{-2}<L_{-4}$, in view of (3.2), we have $I>L_{-2}$, which is a contradiction, and so $L_{-2} \geq L_{-4}$. From (3.1), we obtain

$$
I=\frac{L_{-2}+L_{-3}}{\left(L_{-3}+L_{-4}\right) /\left(L_{-2}+L_{-4}\right)+L_{-3}} \geq \frac{I+L_{-3}}{\left(I+L_{-3}\right) / 2 I+L_{-3}} \geq \frac{I+S}{(I+S) / 2 I+S}=I
$$

which implies that $L_{-3}=S$. In addition,

$$
I=\frac{L_{-2}+S}{L_{-1}+S} \geq \frac{I+S}{L_{-1}+S}=\frac{2 I S}{L_{-1}+S}
$$

and so $L_{-1}=S$ and $L_{-2}=I$. The proof is complete.

LeMma 3.7. Let $\left\{x_{n}\right\}_{n=-2}^{\infty}$ be a positive oscillatory solution of (3.1) for which there exists $N \geq 0$ such that

$$
x_{N-1}>1>x_{N-2}, x_{N}>\frac{1}{2} .
$$

Let (2.4) hold and let $L_{0}$ be any accumulation point for $\left\{x_{n}\right\}_{n=-2}^{\infty}$. Then

$$
L_{0} \in\{I, S\}
$$

Proof. For the sake of contradiction, suppose that

$$
L_{0} \in(I, S) \text {. }
$$

Then there exists a subsequence $\left\{n_{i}\right\}_{i=1}^{\infty}$ such that

$$
\begin{gathered}
\lim _{i \rightarrow \infty} x_{n_{i}}=L_{0}, \\
\lim _{i \rightarrow \infty} x_{n_{i}+k}=L_{k} \in[I, S], \quad k=-6,-5,-4,-3,-2,-1,0 .
\end{gathered}
$$

Assume that $L_{-1} \in\{I, S\}$. In view of Lemma 3.6, we have

$$
L_{-3}=L_{-5}=L_{-1}, \quad L_{-2}=L_{-4}=\frac{L_{-1}}{2 L_{-1}-1}
$$

and so $L_{0} \in\{I, S\}$, which is a contradiction. Therefore, $L_{0}, L_{-1}, L_{-2} \in(I, S)$. There exists $\epsilon>0$ such that

$$
L_{0}, L_{-1}, L_{-2} \in\left(I+2 \epsilon, \frac{I+2 \epsilon}{2(I+2 \epsilon)-1}\right)
$$

and an integer $N>0$ such that

$$
x_{n_{N}-2}, x_{n_{N}-1}, x_{n_{N}} \in\left[I+\epsilon, \frac{I+\epsilon}{2(I+\epsilon)-1}\right] .
$$


Proceeding as in the proof of Theorem 3.5, we have

$$
x_{n} \in\left[I+\epsilon, \frac{I+\epsilon}{2(I+\epsilon)-1}\right] \text { for } n \geq n_{N},
$$

which implies that $I \geq I+\epsilon$, which is a contradiction. The proof is complete.

Theorem 3.8. Let $\left\{x_{n}\right\}_{n=-2}^{\infty}$ be a positive oscillatory solution of (3.1) for which there exists $N \geq 0$ such that $x_{N-1}>1>x_{N-2}, x_{N}>1 / 2$. Then

$$
\lim _{n \rightarrow \infty} x_{2 n}, \quad \lim _{n \rightarrow \infty} x_{2 n+1}
$$

exist and they are finite.

Proof. From Theorem 3.5 the solution $\left\{x_{n}\right\}_{n=-2}^{\infty}$ is bounded from above and below. Let (2.4) hold. If $I=S$, the solution $\left\{x_{n}\right\}_{n=-2}^{\infty}$ of (3.1) is convergent and there is nothing to prove. Let $I<S$. Then $I<1<S$. In addition, and with the use of Lemma 3.7, there exists a subsequence $\left\{n_{i}\right\}_{i=1}^{\infty}$ such that

$$
\begin{array}{ll}
\lim _{i \rightarrow \infty} x_{n_{i}+k}=S, & k=-4,-2,0, \\
\lim _{i \rightarrow \infty} x_{n_{i}+k}=I, & k=-3,-1 .
\end{array}
$$

Therefore, there exists $N$ such that

$$
x_{N-1}<1<x_{N-2}, x_{N}
$$

From (3.1) and Lemma 3.3, we have

$$
x_{N+2 k-1}<1<x_{N+2 k}, \quad k=0,1, \ldots
$$

Let $L_{i}$, where $i=-1,0$, be arbitrary accumulation points for the subsequences $\left\{x_{N+2 k+i}\right\}_{k=0}^{\infty}$. Then $L_{-1} \leq 1 \leq L_{0}$. In view of Lemma 3.7, we have $L_{-1}=I$ and $L_{0}=S$. The proof of Theorem 3.8 is complete.

We end this section with the following open problem.

Open problem 3.9. Prove or disprove the existence of unbounded solutions of (3.1).

\section{Existence of unbounded solutions of (1.1)}

In this section, we show that if $\gamma>1$, then (1.1) has unbounded solutions.

Theorem 4.1. Suppose that $\gamma>1$ and let $\left\{x_{n}\right\}_{n=-2}^{\infty}$ be a solution of (1.1) with initial conditions

$$
x_{-2}<\frac{\gamma}{2}, \quad x_{0}<\frac{\gamma}{2}<\frac{\gamma^{2}}{2(\gamma-1)}<x_{-1} \text {. }
$$


Then

$$
\lim _{n \rightarrow \infty} x_{2 n+1}=\infty
$$

Proof. From (1.1), we have

$$
x_{1}=\frac{\gamma x_{-1}+x_{-2}}{x_{0}+x_{-2}} .
$$

In view of (4.1), $\gamma x_{-1}>x_{0}$ and so the expression

$$
\frac{\gamma x_{-1}+x_{-2}}{x_{0}+x_{-2}}
$$

is decreasing in $x_{-2}$ and $x_{0}$. Thus

$$
x_{1}=\frac{\gamma x_{-1}+x_{-2}}{x_{0}+x_{-2}}>\frac{\gamma x_{-1}+\gamma / 2}{\gamma / 2+\gamma / 2}=x_{-1}+\frac{1}{2} .
$$

Also, in view of (4.1), we have

$$
\gamma x_{-1}>x_{-1}+\frac{\gamma^{2}}{2}>x_{-1}+\gamma x_{0} .
$$

Thus

$$
\gamma>\frac{x_{-1}+\gamma x_{0}}{x_{-1}}
$$

and so

$$
\frac{\gamma}{2}>\frac{x_{-1}+\gamma x_{0}}{2 x_{-1}}=\frac{x_{-1}+\gamma x_{0}}{x_{-1}+x_{-1}}>\frac{x_{-1}+\gamma x_{0}}{x_{-1}+x_{1}}=x_{2} .
$$

Inductively, it follows that for $n=0,1, \ldots$,

$$
x_{2 n}<\frac{\gamma}{2}, \quad x_{2 n+1}>x_{2 n-1}+\frac{1}{2} .
$$

Thus

$$
\lim _{n \rightarrow \infty} x_{2 n+1}=\infty
$$

The proof is complete.

We end with the following open problem.

Open problem 4.2. Suppose that the initial values $x_{-2}, x_{-1}, x_{0}$ of (1.1) are chosen so that

$$
0<x_{-2}<\frac{\gamma}{2}, \quad 0<x_{0}<\frac{\gamma}{2}<\frac{\gamma^{2}}{2(\gamma-1)}<x_{-1} .
$$


Show that the following results hold:

(a) if $1<\gamma<2$, then

$$
\lim _{n \rightarrow \infty} x_{2 n}=1-\frac{\gamma}{2}
$$

(b) if $\gamma \geq 2$, then

$$
\lim _{n \rightarrow \infty} x_{2 n}=0
$$

\section{References}

[1] E. Camouzis, C. H. Gibbons, and G. Ladas, On period-two convergence in rational equations, J. Difference Equ. Appl. 9 (2003), no. 5, 535-540.

[2] M. R. S. Kulenović and G. Ladas, Dynamics of Second Order Rational Difference Equations. With Open Problems and Conjectures, Chapman \& Hall/CRC, Florida, 2002.

E. Camouzis: Department of Mathematics, Deree College, The American College of Greece, Aghia Paraskevi, 15342 Athens, Greece

E-mail address: camouzis@acgmail.gr

R. DeVault: Department of Mathematics, Northwestern State University of Louisiana, Natchitoches, LA 71497, USA

E-mail address: rich@nsula.edu

G. Papaschinopoulos: Department of Electrical and Computer Engineering, Democritus University of Thrace, 67100 Xanthi, Greece 